\title{
An Automatic Goodness Index to Measure Fingerprint Minutiae Quality
}

\author{
Edel García Reyes, José Luis Gil Rodríguez, and Mabel Iglesias Ham \\ Advanced Technologies Application Center, \\ 7a \#21812 e/ 218 y 222, Rpto. Siboney, Playa. C.P. 12200, \\ Ciudad de La Habana, Cuba \\ Office Phone number: $(+) 537.271 .4787$ \\ Fax number: $(+) 537.272 .1667$ \\ \{egarcia, jlgil, miglesias\} @cenatav.co.cu
}

\begin{abstract}
In this paper, we propose an automatic approach to measure the minutiae quality. When image of $500 \mathrm{dpi}$ is captured, immediately the enhancement, thinning and minutiae extraction processes are executed. The basic idea is to detect the spatial $\beta_{0}$ - Connected minutiae cluster using the Euclidean distance and quantify the number of element for each group. In general, we observe that more than five element in a group is a clue to mark all points in the cluster as bad minutiae. We divide the image in block of $20 \times 20$ pixels. If one block contains bad minutiae it is mark as a bad block. The goodness quality index is calculated as the proportion of bad blocks respect to the number of total blocks. The proposed index was tested on the FVC2000 fingerprint image database.
\end{abstract}

\section{Introduction}

When some agency face the task of make a massive load of card ink fingerprint, to create a large data base, it is necessary to put maximum care in the quality of fingerprint images that will feed the Automatic Fingerprint Identification System (AFIS). It is known that performance of an AFIS relies heavily on the quality of input fingerprint images. Although, it is normal to have a manual quality control, it is desirable that system automatically reject the bad fingerprint that do not accomplish the quality threshold. If each image is storage, with its quality measure associated, it is possible to calculate the average database quality.

Several methods for measuring the quality of fingerprint images were found in the literature [1,2]. In general, they can divide in five categories: methods using standard deviation, methods using directional contrast, methods using Gabor features, methods using local ridge structure, and methods using Fourier spectrum.

The completely path to make fingerprints matching can be segmented in three moments: early steps, middle steps, and last steps. The early steps correspond to the image enhancement and binarization; the middle is associated to thinning, skeleton reconstruction process and the minutiae detection. The last steps in our sequence are the graph based representation of the fingerprint and finally, the graph matching and visual verification. Minutiae extraction corresponds to the middle steps. The basic 
idea is evaluate the quality in the middle step in this sequence. We propose to detect spatial minutiae clusters using the Euclidean distance and quantify the number of element for each group. In general, the idea is to consider high spatial density of minutiae as a sign of not good automatically minutiae detection. We observe that more than five element in a group is a clue to mark all points in the cluster as bad minutiae. We divide the image in block of $20 \times 20$ pixels. If one block contains a bad minutiae it is marked as a bad block. The goodness quality index is calculated as the proportion of bad blocks respect to the number of total blocks. With our approach the Goodness index is possible to use it in both sense to evaluate the image enhancement algorithm and the expert visual evaluation of image. The global fingerprint's database quality is the average of all image's quality introduced in it.

The remainder of the paper is organized as follows: Section 2 presents our global workflow to create fingerprint database from card ink impression. Section 3 describes the spatial minutiae cluster algorithm and the goodness quality index. The descriptions of our experiments and results are showed in section 4. Finally, conclusions are presented in section 5.

\section{Global Workflow to Create Fingerprint Database from Card Ink Impression}

When we have a lot of card ink impression paper that we need to covert in digital format, in order to work with an Automatic Fingerprint Identification System, is very important to guarantee the database quality. This may determine the acceptation of the system by the staff of forensic expert personal.

Load one million of card ink paper may take around six months. This is a great and determinant effort that must be carefully organized, and appropriated software tools are required to reach the quality in the minimum possible time.

First, it is necessary to have an automatic tool to massive scanning of card ink papers with the possibility of an automatic and manual cut of the frames including the image of each finger. Every fingerprint model must have a barcode associated. In this way, the code in the moment that it is scanned is recognized and used as an identifier in the filename of each fingerprint image.

After the cut, it is necessary apply a mask to segment the image in regions which correspond to foreground and background, then a second cut is performed to optimize the followings processes. Immediately, the image enhancement, binarization, thinning, skeleton reconstruction and minutiae extraction are executed. We know that these processes are computational intensive, fundamentally the image enhancement. We enter in a trade of between the calculus time and the quality of the minutiae set.

In this moment, a first calculus of the goodness index is made before to pass the image to visual quality control. The system highlights that finger minutiae set is not reaching the quality threshold. Then, it is possible to examine the automatic cut quality and the quality of minutiae set. May be, some image need a manual cut and edition where some spurious minutiae must be deleted and mark new ones. So, it is necessary a minutiae editor tool. It is the human expert who finally has the responsibility to decide if one fingerprint image could be storage in the database. A second goodness quality index must be calculated to be storage associated to the 
image in the database after the manual edition. Together with the image and the goodness index, the graph based representation obtained from the minutiae set is also stored.

It is possible to have various scanners to read card ink papers to some repository and other work stations to processing the images. A job manager may distribute the digital images of card models to the processing stations following some priorities. The job manager always has the possibility to examine the average quality of the images stored by a determined operator, or evaluate the average quality of whole database in some determined period of time.

\section{Spatial Minutiae Clustering and Goodness Quality Index}

In general, the idea is to consider high spatial density of minutiae as a sign of not good automatically minutiae detection. We need to look for the minutiae clusters on the fingerprint image and evaluate its spatial concentration to decide if there is a cluster of bad minutiae. In some cases, when there are singular points like core or delta, it is possible to observe some natural aggregation of point on the fingerprint. In that situation our approach underestimate the fingerprint quality. However, in general, a minutiae cluster is associated with a region of bad image quality where the enhancement algorithm has a bad performance, and the ridge pattern obtained is false. Then, the feature extraction algorithm detect many spurious points near each other.

\subsection{Spatial Minutiae Clustering}

Every minutia has associated its coordinate $x$ and $y$. To find spatial minutiae clusters based on its coordinates, it is possible to use an algorithm that operate in a metric space, using a similarity function based on the Euclidean distance. Algorithms Leader, K-Means, and ISODATA [4] were evaluated and rejected. All of them may obtain different solutions on different order on the input data. In this case, when an impression suffers some geometrical transformation the feature points may change its order. Almost all these algorithms need to know the number of cluster to obtain. Precisely, the goodness quality index proposed is based on the cardinality of the clusters. We need to detect natural points clusters. If a number of cluster if defined previously, all minutiae are distribute between these groups and the membership of some cluster may grow artificially. We prefer an algorithm to detect small connected minutiae groups, regardless the number of cluster obtained.

It is necessary to use some algorithm that detects automatically the number of minutiae clusters in input set. Besides, we are looking for clusters that no necessarily follow an elliptical shape. With these elements in mind, we think about using some algorithm to detect connected component [3].

\subsection{1 $\beta_{0}$-Connected Component}

Let $\mathrm{O}=\left\{\mathrm{O}_{1}, \ldots, \mathrm{O}_{\mathrm{m}}\right\}$ be set of objects, $\beta$ a similarity function and $\beta_{0}$ similarity threshold for $\beta$. 


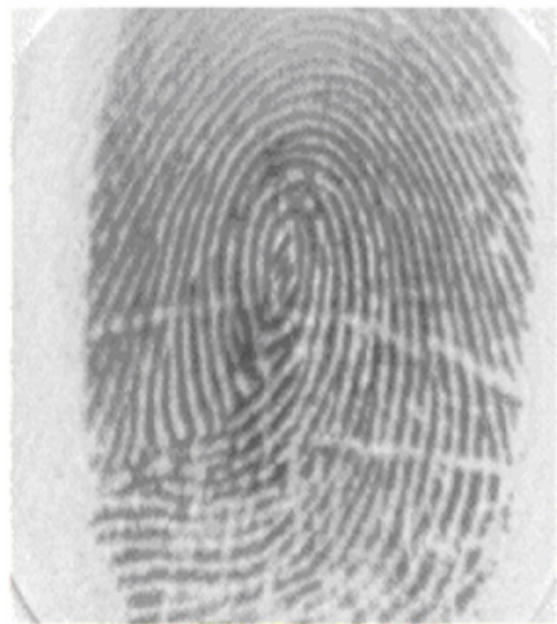

a)

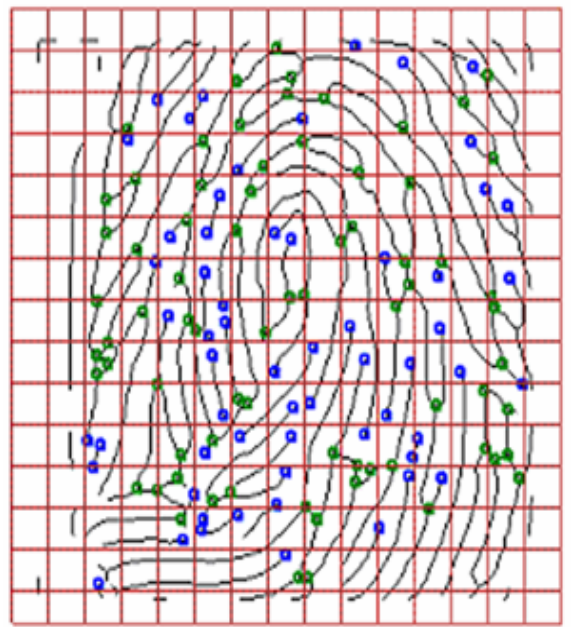

c)

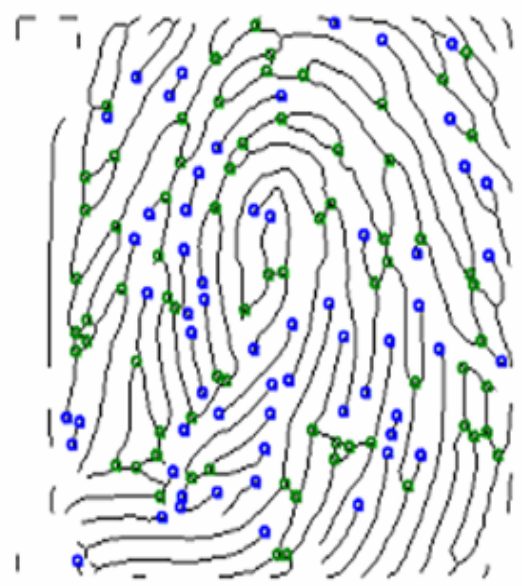

b]

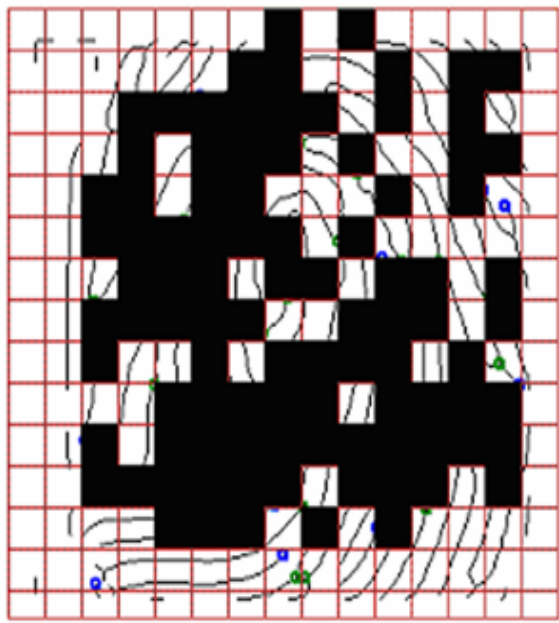

d)

Fig. 1. Goodness quality index $=0.63$. a) Bad image from FVC2000 database. b) Minutiae detection. c) Division in block of 20 x 20 pixels. d) Bad block detection.

Definition 1. Two object $\mathrm{O}_{\mathrm{i}}, \mathrm{O}_{\mathrm{j}}$ are denominated $\beta_{0}$ similar if $\beta\left(\mathrm{O}_{\mathrm{i}}, \mathrm{O}_{\mathrm{j}}\right) \geq \beta_{0}$.

Definition 2. Let $S \subseteq \mathrm{O}, \mathrm{S} \neq \varnothing$ be $\beta_{0}$-Connected set respect to $\beta$ iff $\forall \mathrm{O}_{\mathrm{i}}, \mathrm{O}_{\mathrm{j}} \in \mathrm{S}$, $\exists\left\{\mathbf{O}_{\mathrm{S} 1}, \mathbf{O}_{\mathrm{S} 2}, \ldots, \mathrm{O}_{\mathrm{St}}\right\}$ such that $\mathrm{O}_{\mathrm{i}}=\mathrm{O}_{\mathrm{S} 1}, \mathrm{O}_{\mathrm{St}}=\mathrm{O}_{\mathrm{j}}$ and $\beta\left(\mathrm{O}_{\mathrm{Si}-1}, \mathrm{O}_{\mathrm{Si}}\right) \geq \beta_{0}, \mathrm{i}=2,3, \ldots, \mathrm{t}$.

It means that for any pair of point in $S$ there is a succession of elements in $S$ beginning in $\mathrm{O}_{\mathrm{i}}$ and finishing in $\mathrm{O}_{\mathrm{j}}$ such that one is $\beta_{0}$ similar to the next. 
FindConnectedSet (PairEquivalencelist, in) \{

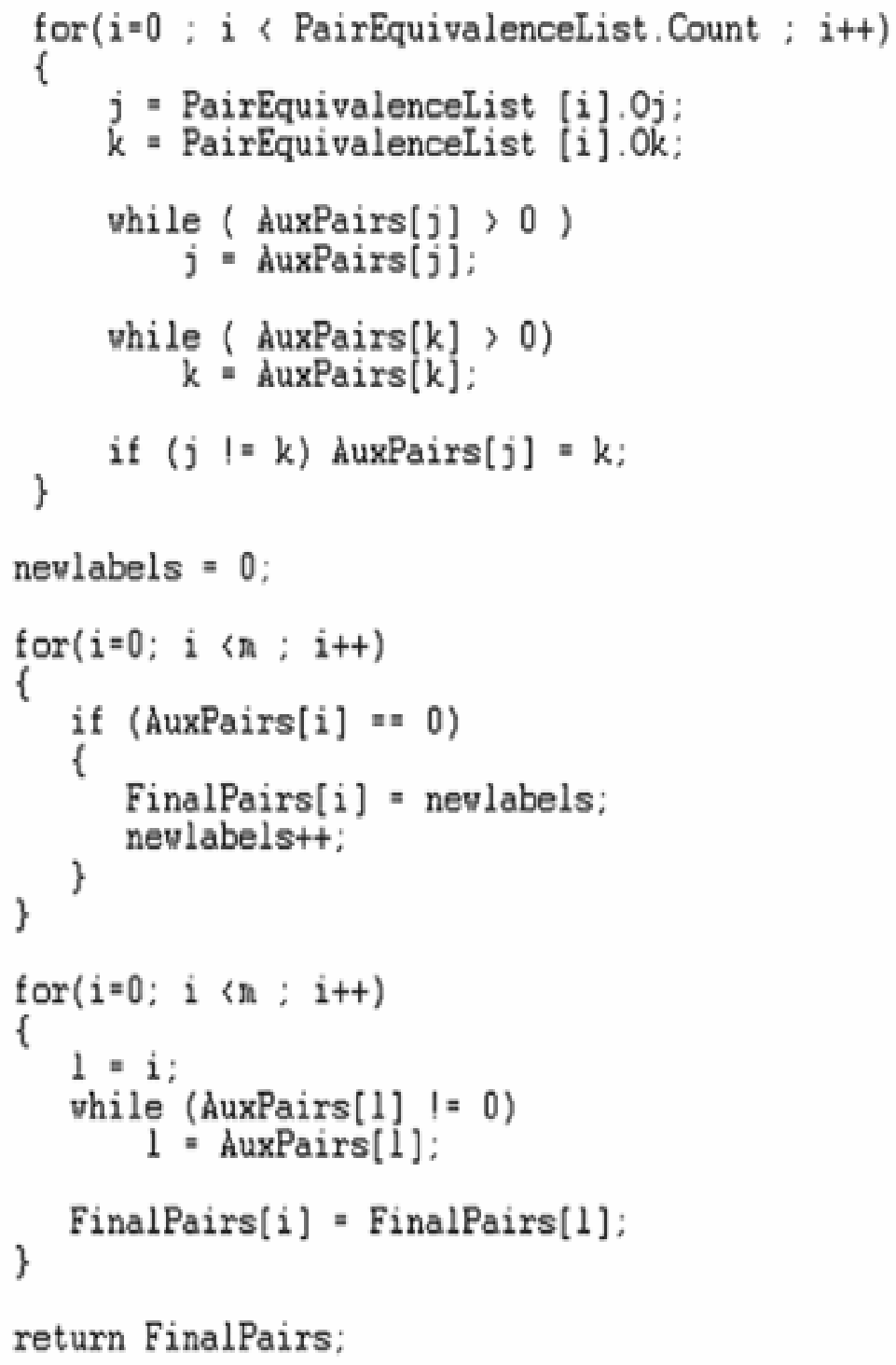

Fig. 2. The pseudocode of the main step of the proposed algorithm 


\subsubsection{Algorithm}

Our algorithm to detect $\beta_{0}$-Connected sets is used to observe if there are spatial clusters of minutiae in the image. This algorithm partitions a set of $m$ minutiae into $k$ disjoint $\beta_{0}$-Connected clusters. A similarity function $\beta$ that compute the inverse of distance between minutiae is assumed along with a predefined similarly threshold value, $\beta_{0}$.

The algorithm proceeds as follow for a minutiae set $O$ and $\beta_{0}$ threshold. $\beta$ is the inverse of Euclidean distance:

PairEquivalenceList $=$ FindPairNearMinutiae $\left(O, \beta, \beta_{0}\right)$
$\beta_{0}$-ConnectedSet $=$ FindConnectedSet $($ PairEquivalenceList, $\mathrm{m})$

To find the equivalence pairs is the similar process to build the adjacency matrix of a graph. A pair of minutiae is included in the PairEquivalenceList if they are $\beta_{0}$ similar.

Immediately, the equivalence pair list is processed to obtain the $\beta_{0}$-Connected set following the flow showed in figure 2. For our experiments we found 0.04 as a good threshold for $\beta_{0}$.

\subsection{Goodness Quality Index}

We use other threshold to classify a cluster as a bad cluster or good cluster. This threshold is related with the number of element in the cluster. If the cluster has more than 5 minutiae it is marked as bad cluster. Then, we divide the fingerprint images in blocks of $20 \times 20$ pixels. A block is considered to have bad quality if it contains at less one minutia belonging to a bad cluster and otherwise it is considered a good block.

We defined a goodness quality index $(G q I)$ :

$$
G q I=1-\frac{\text { number of bad quality bocks }}{\text { number of blocks }} .
$$

\section{Experiments and Results}

The algorithm described has been implemented and tested. FVC2000 database was used to obtain the quality measure thresholds. FVC2000 is a public database collected using optical sensing techniques. The experts performed a visual preselection of four groups of fingerprint images categorized in: good, bad, smudged and dried fingerprint image.

The minutia sets obtained for each fingerprint image were analysed and categorized as good, regular or bad by the experts.

We have found the following quality thresholds (table 1): 
Table 1. The thresholds of quality

\begin{tabular}{|c|c|c|}
\hline \multicolumn{2}{|c|}{ Thresholds } & Description \\
\hline GqI $<$ & 0.78 & Bad minutiae set \\
\hline $0.78 \leq$ & GqI $<0.85$ & Regular minutiae set \\
\hline GqI $\geq$ & 0.85 & Good minutiae set \\
\hline
\end{tabular}

The following results (table 2) show the performance of the goodness minutiae index for each fingerprint image category:

Table 2. Goodness quality index for each fingerprint image group from FVC2000 database

\begin{tabular}{|c|l|l|l|l|c|}
\hline & Good & Bad & Smudged & Dried & Total \\
\hline $\begin{array}{c}\text { Number of } \\
\text { image }\end{array}$ & 120 & 40 & 68 & 92 & 320 \\
\hline $\begin{array}{c}\text { Average } \\
\text { GqI }\end{array}$ & 0.93 & 0.78 & 0.87 & 0.90 & 0.89 \\
\hline
\end{tabular}

We observed that the goodness quality index not always is consistent with visual human assessment, because our image enhancement and feature detection algorithms are robust and perform very well to obtain the minutiae set, even in some image where there are scars and some level of noise.

We noted that, according to our approach, the FVC2000 database has a goodness quality index of 0.89 relative to our minutiae detection algorithm.

On another hand, the experts made a manual minutia edition of the fingerprint image included in the bad group $(\mathrm{GqI}=0.78)$ and after that it was recalculated the goodness quality index. In this case, the averaged value was elevated to 0.98 . It shows that our goodness quality index reach a value near 1 when a good minutiae set is obtained.

\section{Conclusion}

In this paper we present an approach to measure the minutiae quality that guarantee a control about the average quality of a fingerprint image database.

In general, we observed that our heuristic detect more true bad cluster than false bad cluster. From this point of view it follow a pessimist strategy, because it is possible that an image was stored in the database with some block marked as bad when in reality there was a minutiae concentration due a singularity fingerprint ridge flow.

\section{References}

1. Yi-Sheng, M., Patanki, S., Hass, N.: Fingerprint Quality Assessment. Automatic Fingerprint Recognition Systems, Chapter 3 , Ratha, N., and R. Bolle, Editors. Springer-Verlag (2004) 55-66 
2. Vallarino, G., Gianarelli, G., Barattini, J., Gómez, A., Férnandez, A., Pardo, A.: Performance Improvement in a Fingerprint Classification System Using Anisotropic Diffusion. Lecture Notes in Computer Science, Springer-Verlag, No. 3287. (2004) 582-588

3. Martínez, F., Ruiz, J., Lazo, M.: Structuralization of Universes. Fuzzy Set and System, Vol. 112/3. (2000) 485-500.

4. Hartigan, J. A.: Clustering Algorithms, New York: John Wiley and Sons (1975) 